\title{
Dual Ion Species Plasma Expansion from Isotopically Layered Cryogenic Targets
}

\author{
G. G. Scott,,${ }^{1,}$ D. C. Carroll,${ }^{1}$ S. Astbury,${ }^{1}$ R. J. Clarke, ${ }^{1}$ C. Hernandez-Gomez,${ }^{1}$ M. King, ${ }^{2}$ A. Alejo, ${ }^{3}$ \\ I. Y. Arteaga, ${ }^{1}$ R. J. Dance, ${ }^{2}$ A. Higginson, ${ }^{2}$ S. Hook, ${ }^{1}$ G. Liao, ${ }^{4}$ H. Liu, ${ }^{1,5,6}$ S. R. Mirfayzi, ${ }^{3}$ D. R. Rusby, ${ }^{1,2}$ \\ M. P. Selwood, ${ }^{1}$ C. Spindloe, ${ }^{1}$ M. K. Tolley, ${ }^{1}$ F. Wagner, ${ }^{7}$ E. Zemaityte ${ }^{1,2}$ M. Borghesi, ${ }^{3}$ S. Kar, ${ }^{3,1}$ \\ Y. Li, ${ }^{5,6}$ M. Roth, ${ }^{8}$ P. McKenna, ${ }^{2}$ and D. Neely ${ }^{1,2, \dagger}$ \\ ${ }^{1}$ Central Laser Facility, STFC Rutherford Appleton Laboratory, Didcot OX11 OQX, United Kingdom \\ ${ }^{2}$ Department of Physics SUPA, University of Strathclyde, Glasgow G4 ONG, United Kingdom \\ ${ }^{3}$ Department of Pure and Applied Physics, Queen's University of Belfast, Belfast BT7 1NN, United Kingdom \\ ${ }^{4}$ Key Laboratory for Laser Plasmas (MoE) and Department of Physics and Astronomy, Shanghai Jiao Tong University, \\ Shanghai 200240, China \\ ${ }^{5}$ Beijing National Laboratory for Condensed Matter Physics, Institute of Physics, CAS, Beijing 100190, China \\ ${ }^{6}$ School of Physical Sciences, University of Chinese Academy of Sciences, Beijing 100049, China \\ ${ }^{7}$ PHELIX group, GSI Helmholtzzentrum für Schwerionenforschung GmbH, Darmstadt 64291, Germany \\ ${ }^{8}$ Fachbereich Physik, Technische Universität Darmstadt, Darmstadt 64289, Germany
}

(Received 27 September 2017; revised manuscript received 19 February 2018; published 18 May 2018)

A dual ion species plasma expansion scheme from a novel target structure is introduced, in which a nanometer-thick layer of pure deuterium exists as a buffer species at the target-vacuum interface of a hydrogen plasma. Modeling shows that by controlling the deuterium layer thickness, a composite $\mathrm{H}^{+} / \mathrm{D}^{+}$ ion beam can be produced by target normal sheath acceleration (TNSA), with an adjustable ratio of ion densities, as high energy proton acceleration is suppressed by the acceleration of a spectrally peaked deuteron beam. Particle in cell modeling shows that a $(4.3 \pm 0.7) \mathrm{MeV}$ per nucleon deuteron beam is accelerated, in a directional cone of half angle $9^{\circ}$. Experimentally, this was investigated using state of the art cryogenic targetry and a spectrally peaked deuteron beam of $(3.4 \pm 0.7) \mathrm{MeV}$ per nucleon was measured in a cone of half angle $7^{\circ}-9^{\circ}$, while maintaining a significant TNSA proton component.

DOI: 10.1103/PhysRevLett.120.204801

Laser driven ion acceleration is the subject of intense study as the unique characteristics of the energetic ion beams have made them candidates for many potential applications [1,2]. The most widely investigated acceleration mechanism is target normal sheath acceleration [3] and the pertinent features of TNSA accelerated beams are reproduced by analytic plasma expansion models [4-7]. These ion beams have an inherently broad, thermal energy distribution whereas many potential applications of these compact sources require some degree of spectral control. This is motivating the investigation of new approaches to control the ion energy distribution at the source. Analytic models have shown that interactions between two or more ion species during plasma expansion can significantly modify the final ion spectral distribution [8-12]. The specific changes to the spectra depend on the ions' spatial distribution within the plasma and this provides an approach to achieving spectral control.

Published by the American Physical Society under the terms of the Creative Commons Attribution 4.0 International license. Further distribution of this work must maintain attribution to the author(s) and the published article's title, journal citation, and DOI.
In a target comprised of two ion species, the species with the higher charge to mass ratio, $Q / A$, is accelerated faster. Therefore, if the light ion species exists as a thin layer, it typically decompresses and expands away from the heavier one [8,12-15]. This results in most of the lighter ion species being subject to the same electrostatic field, and the acceleration of a quasimonoenergetic light ion distribution. An electrostatic field structure can also form at the interface of two ion species $[9,16,17]$, further accelerating the lighter ion species. Quasimonoenergetic deuterons have been accelerated via this mechanism, with the deuterons existing as the lighter ion species in a mass limited $\mathrm{D}_{2} \mathrm{O}$ target $[9,16]$. The different rates of expansion of two ion species have also been exploited in double pulse interactions, in which a prepulse preexpands a layer of hydrogen; this tenuous plasma subsequently undergoes acceleration later in time by the field generated by a higher intensity pulse, resulting in quasimonoenergetic proton acceleration [18]. Also, when ultrathin foils expand to the extent that relativistic induced transparency occurs, volumetric laser heating of electrons can provide a boost to the heavier ion expansion rate, which in turn modifies the lighter ions' energy spectrum $[19,20]$ and beam profile [21]. In each of these schemes, the expanding heavier ions strongly affect 

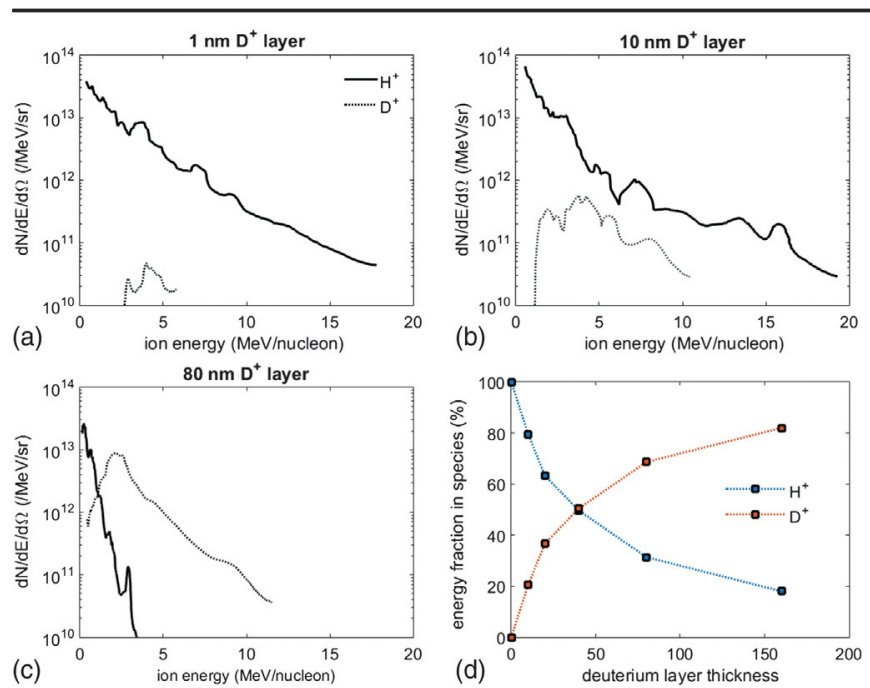

FIG. 1. (a)-(c) Simulated proton and deuteron spectra from targets with different deuterium thicknesses on the rear of a hydrogen plasma. (d) The fraction of total ion beam energy carried by each species as a function of the deuterium layer thickness.

the properties of the lighter ion beam, while the heavier ions are effectively tamped by the lighter ions.

In this Letter, we present the first theoretical consideration and experimental demonstration of a plasma expansion scheme where the heavier ion species exists as a separate layer between the target vacuum interface and a lighter ion species. In this configuration, light ions strongly affect the heavier ion population expansion dynamics, resulting in a spectrally peaked, directional beam of heavier ions. It is shown that the fraction of total ion beam energy in each species and their spectral features can be controlled by varying the heavier ion layer thickness. Experimentally, this is achieved using state of the art cryogenic targetry [22], where nanometer-thick layers of solid density deuterium are frozen onto the rear surface of a target substrate, on which hydrogen rich monolayers are present.

To investigate this plasma expansion scheme, 1D PIC modeling was conducted using the EPOCH code [23]. A $25 \mu \mathrm{m}$ grid, resolved by $10^{5}$ cells, was populated with a $2 \mu \mathrm{m}$ thick proton species and a variable thickness deuteron species at the target rear, both with a maximum density of 51.4 times the critical density and quasineutralized by electrons. A laser of intensity profile $I(t)=I_{0} \exp \left(-(t / \tau)^{2}\right)$ irradiates the target, where $I_{0}=2 \times 10^{19} \mathrm{~W} \mathrm{~cm}^{-2}, \tau=680 \mathrm{fs}$ and $\lambda=1.054 \mu \mathrm{m}$.

Figure 1 shows the ion spectra obtained in the laser forward direction. The results show a clear dependence on the deuterium layer thickness. For a $1 \mathrm{~nm}$ layer, deuterons are observed with an average energy of $4.3 \mathrm{MeV} /$ nucleon and with a spectral peak, characterized by a variance of $0.7 \mathrm{MeV}$. The presence of such a thin layer has a minimal effect on the TNSA protons, which are found to be accelerated to a similar maximum energy and only slightly reduced number as in the test case where no deuterium layer is present.

The accelerating field across a $1 \mathrm{~nm}$ deuterium layer is relatively uniform. This results in a low energy divergence of the accelerated deuterium in the early stage of the simulation. Consequently, a significant accelerating field extends through the decompressed deuterium layer to the proton density front. With twice the deuteron $Q / A$, protons are accelerated beyond the deuteron density front, and as the proton density front breaks that of the deuterons, the deuterons are shielded from further acceleration by the electric field, and protons dominate the acceleration at later times. If the layer is sufficiently thin, the short breaking period means that the deuterons are subject to uniform electric field during this phase. The result is a deuteron beam with a preserved spectral peak, with an average energy proportional to both the initial field strength and the time this field acts upon it, as determined by the proton breaking time.

As the deuterium thickness is increased, both the deuteron beam energy spread and maximum energy are increased, which is consistent with this interpretation. The accelerating field magnitude at the proton front is diminished as it extends through the thicker deuteron layers. This leads to a lower energy proton beam acceleration, and an increase in the breaking time as the slower protons transit the thicker deuteron layer. This leads to a spatially varying electric field existing over the deuteron front, and hence to a larger energy spread deuteron beam. For increasingly thick layers of deuterium, the mechanism tends towards purely deuteron TNSA.

In this way, the deuteron beam energy spread, as well as the fraction of total ion beam energy carried by each species can be controlled, as shown in Fig. 1(d), simply by controlling the initial deuterium layer thickness.

To ensure that the deuteron spectra result from the bulk hydrogen governing the sheath evolution dynamics at the deuteron front, protons were replaced with a heavier ion species with $Q / A=1 / 6$, and we observe deuterons to be accelerated to $11 \mathrm{MeV} /$ nucleon in a large energy spread thermal spectrum.

Further, we find that the acceleration scheme is robust to the presence of contaminant hydrogen when the deuterium layer is thinner than $10 \mathrm{~nm}$. When an equal density mixture populates the layer, a lower maximum energy, peaked deuteron spectrum is preserved. In this case, the thin $\mathrm{H}^{+} / \mathrm{D}^{+}$composite layer quickly undergoes a density decompression, and has little effect on the sheath evolution at the deuteron front. Again this highlights the importance of the bulk proton expansion for the observed deuteron spectra. For a more detailed discussion of the influence of the target configuration please see the Supplemental Material provided [24].

To investigate the higher dimensionality dynamics, 2D PIC modeling was conducted using the EPOCH code. 


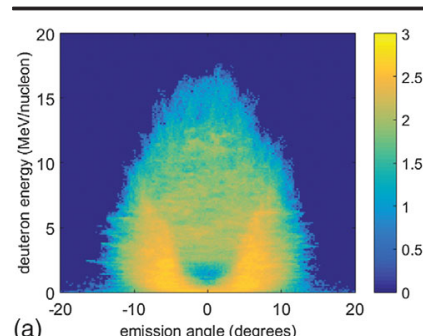

(a)

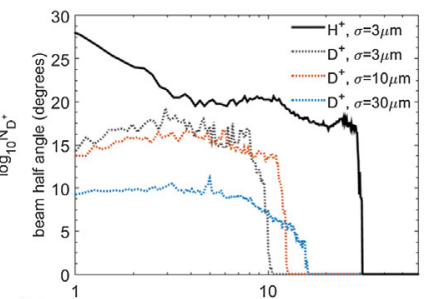

(b)
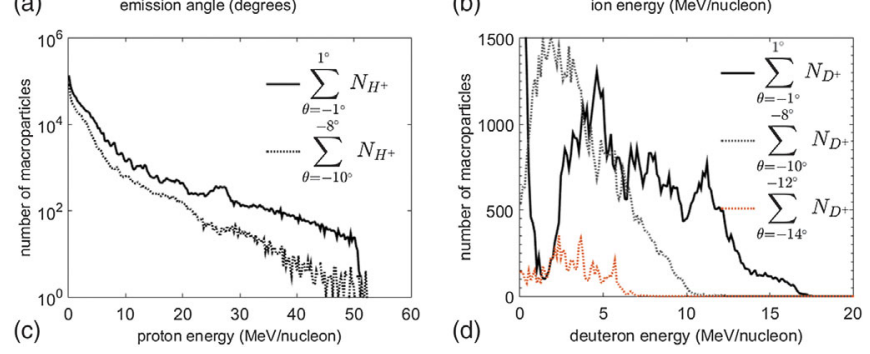

FIG. 2. (a) The angularly resolved deuteron spectrum obtained from 2D PIC simulations, with a $30 \mu \mathrm{m}$ laser spot. (b) The effect of changing the laser spot size on the $1 / e^{2}$ beam half angle. For comparison with experiment, the integrated ion number accelerated at $\theta \pm 1^{\circ}$ is shown for (c) $\mathrm{H}^{+}$for $\theta=0^{\circ}$ and $-9^{\circ}$ (d) $\mathrm{D}^{+}$for $\theta=0^{\circ},-9^{\circ}$, and $-13^{\circ}$.

A laser of the form $I(y, t)=I_{0} \exp \left(-(y / \sigma)^{2}-(t / \tau)^{2}\right)$, with $I_{0}=10^{20} \mathrm{~W} \mathrm{~cm}^{-2}, \tau=300 \mathrm{fs}$ and variable spot size, $\sigma$, irradiates a $3 \mu \mathrm{m}$ thick hydrogen target with a $10 \mathrm{~nm}$ thick deuterium layer to the rear, both with density $51.4 n_{c}$, quasineutralized by electrons. The electron, proton, and deuteron species are represented by 20, 20, and 200 macroparticles per cell, respectively. The simulation box dimensions are $55(x) \times 150(y) \mu \mathrm{m}^{2}$, resolved by a grid of $11000(x) \times 3000(y)$ cells.

Figure 2 summarizes the 2D results and 1D results are reproduced, such as the agreement of the on-axis deuteron spectra, shown in Figs. 1(b) and 2(d). However, an angular dependence of the deuteron spectra is observed, with thermal spectra accelerated off axis as shown in Fig. 2 (a). It is found that the on-axis component of the beam is accelerated in the region where the sheath fields are strongest on the target rear, opposite the FWHM laser field, with the thermal component originating from the weaker sheath regions.

The spot size was therefore varied from $3 \mu \mathrm{m}$ to $30 \mu \mathrm{m}$ to investigate the effect of moving from a strongly $2 \mathrm{D}$ sheath expansion to a more planar expansion, and it was found that with larger spot size, the angular spectrum changes from one dominated by the thermal lobes for a $3 \mu \mathrm{m} \mathrm{spot}$, to one where the spectrally peaked beam becomes a more dominant component for a spot size of $30 \mu \mathrm{m}$. This is a trend expected to continue as the laser spot size increases further. The proton and deuteron spectra at discrete angles are shown in Figs. 2(c) and 2(d) from interactions initiated with a $30 \mu \mathrm{m}$ spot. While the deuteron spectrum shows a strong angular dependence, the proton beam is typical of TNSA acceleration, yielding a thermal spectrum with reduced number and maximum energy as it deviates from the normal.

The deuteron beam is found to be accelerated in a smaller $1 / e^{2}$ half angle cone $\left(\theta_{\frac{1}{2}}\right)$ than that of the proton beam for each spot size investigated, with the maximum $\theta_{\frac{1}{2}}$ of the deuteron beam always being found to approximately match that of the maximum energy protons, as shown in Fig. 2(b).

Experimental TNSA studies of a single species dominated acceleration have consistently shown that the angular width of the accelerated ion species at the detector plane decreases with increasing ion energy [25,26]. This is understood to be a result of the spatially and temporally evolving sheath structure, and the reduced $\theta_{\frac{1}{2}}$ of the deuterium beam is a consequence of the deuterons being accelerated only by the peak field.

Additionally, changing the spot width from $3-30 \mu \mathrm{m}$ is found to have a moderate effect on deuteron $\theta_{\frac{1}{2}}$, where the beam width is reduced from $\theta_{\frac{1}{2}} \approx 15^{\circ}-17^{\circ}$ to $\theta_{\frac{1}{2}} \approx 9^{\circ}$ by initiating a more planar expansion. The $2 \mathrm{D}$ modeling therefore predicts that as the expansion becomes more planar, an increasingly directional, spectrally peaked deuteron beam is accelerated.

The cryogenic targetry system used to conduct the experiment is described extensively elsewhere [22] and was used in this work to cool a $100 \mu \mathrm{m}$ thick, planar gold foil to $7 \mathrm{~K}$. The gold substrate naturally has a significant presence of contaminant monolayers on its surfaces, which is the source of hydrogen in this experiment. A $1 \mathrm{~mm}$ diameter wide, cooled deuterium gas delivering capillary was positioned at a distance of $10 \mathrm{~mm}$ from, and directed toward, the target foil, to ensure uniform coverage over a significant surface area.

A reservoir of deuterium gas with a variable internal pressure of 10-1200 mbar was isolated from the capillary by a solenoid valve, which was opened at $300 \mathrm{~ms}$ and closed at $20 \mathrm{~ms}$ prior to the laser pulse arrival, to freeze deuterium onto the target surface. The short, $20 \mathrm{~ms}$, time is intended to prevent monolayer deposition by target chamber contaminants, the presence of which are minimized via cryogenic vacuum pumping to a chamber pressure of $10^{-6}$ mbar. The deuterium layer thickness was varied by changing the volume of gas introduced to the target via the reservoir pressure.

To confirm the viability of delivering thin deuterium layers via this method, a quartz crystal microbalance target was used for calibration and the deuterium layer thickness was measured to be $7 \pm 2 \mathrm{~nm}$ with a reservoir pressure change of 200 mbar in the test configuration.

The $100 \mu \mathrm{m}$ gold target foil was specifically chosen as electron scattering during the transit of the relatively thick, high $Z$ target ensures a smooth, extended sheath at the target rear, and initiates a planar plasma expansion.

The experiment was conducted using the Vulcan laser [27]. The $1.054 \mu \mathrm{m}$ wavelength laser delivered $(345 \pm 53) \mathrm{J}$ 

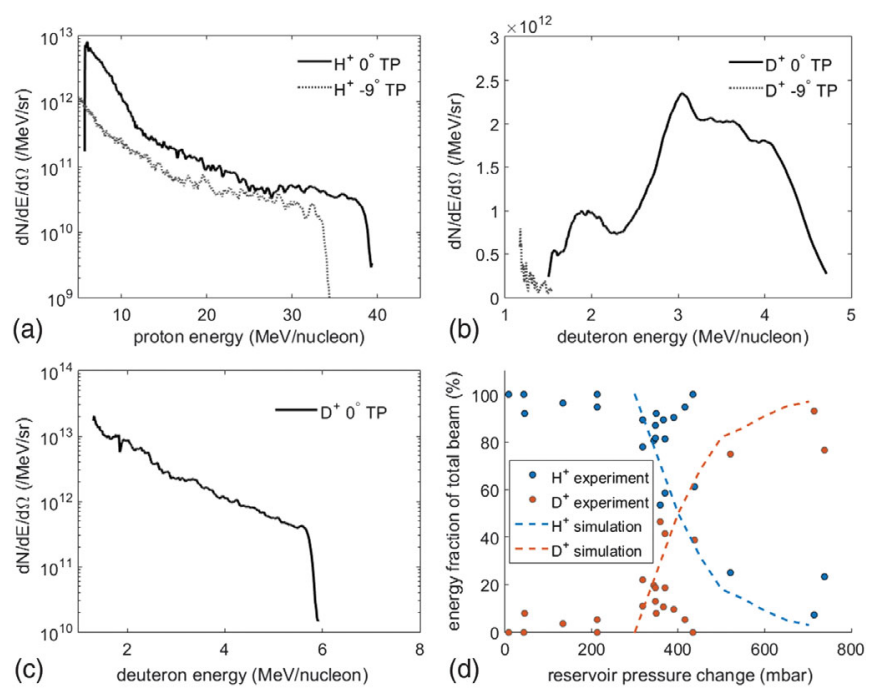

FIG. 3. (a) Proton and (b) deuteron spectra from TPs when operating with a reservoir pressure change of 344 mbar. (c) The spectra obtained for reservoir pressure change of 715 mbar. (d) The fraction of total ion beam energy contained in each species as a function of the reservoir pressure change, which is proportional to the deuterium thickness.

to the target chamber in a pulse duration of $(1.2 \pm 0.3)$ ps. The targets were irradiated at $5^{\circ}$ angle of incidence at best focus with a laser spot FWHM of $5 \mu \mathrm{m}$, giving an average interaction intensity of $10^{20} \mathrm{~W} \mathrm{~cm}^{-2}$. The 300 ps ASE intensity was measured to be $4 \times 10^{-11}$ that of the main pulse.

The ion beam spatial distribution was measured with stacks of $64 \times 25 \mathrm{~mm}^{2}$ sheets of HDv2 radiochromic film, placed $67 \mathrm{~mm}$ from the target rear and $3^{\circ}$ below the interaction plane to allow line of sight for Thomson parabola (TP) ion spectrometers at $0^{\circ}$ (target normal) and $-9^{\circ}$, which sampled a solid angle of $29.6 \mathrm{nsr}$ and $19.8 \mathrm{nsr}$, respectively.

Differential filters were introduced in front of the Fujifilm BAS-TR image plate detector on the TPs. The filter areal density varies with position on the detector magnetic deflection axis, such that $\mathrm{C}^{6+}$ ions are stopped while deuterons pass through, giving an unambiguous deuteron signal [28].

Experimental data are shown in Figs. 3 and 4, and some excellent agreement with numerical modeling is observed. The proton and deuteron spectra in Figs. 3(a) and 3(b), and their angular dependence show very good agreement with PIC modeling shown in Figs. 2(c) and 2(d), where a spectrally peaked deuteron beam is accelerated to $3.4 \mathrm{MeV}$ per nucleon with an energy spread, characterized by a variance of $0.7 \mathrm{MeV}$ per nucleon on the target normal axis.

The raw TP trace and corresponding processed radiochromic film (RCF) dose maps are shown in Fig. 4. On the $-9^{\circ} \mathrm{TP}$, a deuteron signal very close to the noise level was detected, which agrees well with 2D PIC modeling. At $-9^{\circ}$ the PIC modeling suggests that a higher flux deuteron beam
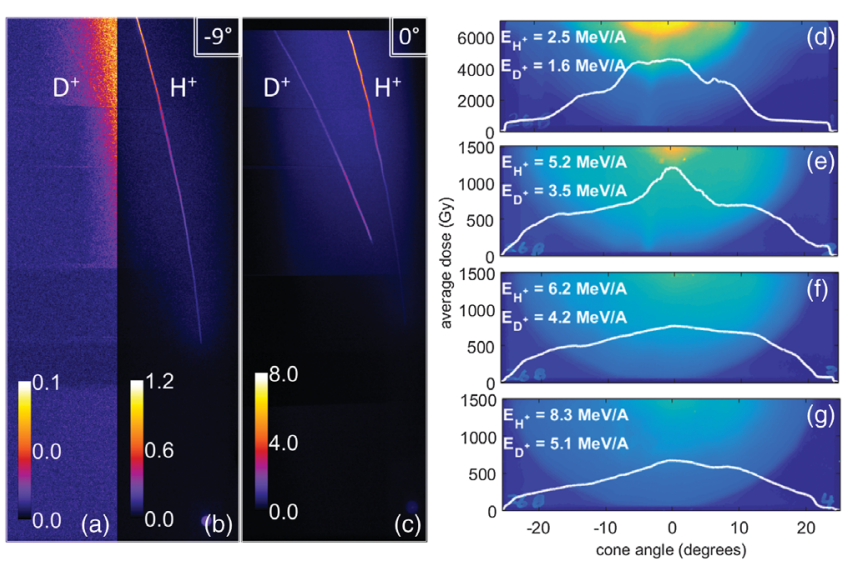

FIG. 4. (a), (b) The $-9^{\circ} \mathrm{TP}$ trace, with electric deflection in the $x$ axis and magnetic deflection in the $y$ axis, split into two regions with different scales (in PSL) to highlight the (a) $\mathrm{D}^{+}$and (b) $\mathrm{H}^{+}$ components. (c) The target normal TP trace. (d)-(g) The beam spatial profile measured by a stacked RCF detector. The average dose measured across the top of each film is plotted in white on each film, the $y$ axis being the scale for this. The color scale range corresponds to the $y$-axis limits for each film.

may be expected, but at only $-13^{\circ}$, PIC modelling predicts a similarly low flux quasithermal beam closer to that observed in experiment. This small deviation is an acceptable error, given its demonstrated dependence on the sheath conditions in the PIC model.

The near absence of deuterons on the $-9^{\circ} \mathrm{TP}$ is clear evidence of a directional beam and is in excellent agreement with 2D PIC modeling shown in Fig. 3(b), and further evidence is observed on the RCF stack detector.

$\mathrm{RCF}$ is sensitive to both protons and deuterons; however, the stopping range of each species is different, and the Bragg peak energy at the corresponding distance into the stack for each film is labeled on Figs. 4(d)-4(g). The film in Fig. 4(g) corresponds to an ion energy only observed for protons on the TP for this shot and therefore provides a proton beam dose profile.

Figures 4(e)-4(g) are virtually identical, except for a centrally peaked high dose feature on Fig. 4(e), with $\theta_{\frac{1}{2}} \approx 3^{\circ}-4^{\circ}$. At $3.5 \mathrm{MeV} /$ nucleon, this deuteron energy almost exactly corresponds to the mean deuteron beam energy, and a similar feature with $\theta_{\frac{1}{2}} \approx 7^{\circ}$ is observed at the lower energy of $2.5 \mathrm{MeV} /$ nucleon.

The RCF sitting below the interaction plane could account for the feature's absence on the $4.2 \mathrm{MeV}$ per nucleon film. Numerical modeling predicts that at energies closer to the cut-off energy, that $\theta_{\frac{1}{2}}$ is reduced, and so the high dose feature may have $\theta_{\frac{1}{2}}<3^{0^{2}}$. Taking account of both $\mathrm{RCF}$ and TP data, compelling evidence for a directional accelerated deuteron beam presents itself.

Figure 3(d) shows a comparison of the pressure change in the deuterium gas reservoir and the fraction of total ion beam energy carried by each species, and a trend similar to that 
predicted by PIC modelling in Fig. 1(d) is obtained. The dashed lines of Fig. 3(d) are a simple linear relationship, $\Delta P=t_{D} x+c$, fitted with the PIC modelling conversion efficiency data of Fig. 1(d), and are shown as a guide between reservoir pressure change, $\Delta P$, to the deuterium thickness, $t_{D}$.

Finally, Fig. 3(c) shows that a high purity deuteron beam can be accelerated using this experimental setup, where more than $10^{13} \mathrm{MeV}$ deuterons/sr were accelerated, representing $93 \%$ of the total beam energy, tending towards a single species dominated, deuteron TNSA. This represents a conversion efficiency of $0.5 \%$ of laser energy into deuterons, and when correcting for laser [29] and target [30-32] parameter differences, is comparable to a similar technique where deuterated water was used to accelerate pure deuteron beams [33].

To conclude, a novel plasma expansion scheme has been investigated where a lower $Q / A$ ion species exists at the target rear vacuum interface, originally acting as a buffer between the teravolt sheath fields and a higher $Q / A$ ion. This unique target configuration has been demonstrated to offer spectral and angular divergence control of the heavy ion beam and global energy partitioning between ion species, and experimental results are shown to give excellent agreement with numerical modeling of the beam characteristics.

Numerically, important parameters have been identified for control of the mechanism, such as the mean deuteron energy dependence on the proton breaking time and peak field strength; the deuteron beam angular spectral distribution was also shown to be dependent on the laser spot size, a parameter within experimental control.

With facilities worldwide planning [34], or actively investigating fast ignition [35-37], we foresee this work to be of relevance. Maxwellian proton $[38,39]$ and deuteron [40] distributions have similarly useful energy ranges for fast ignition of $1-27 \mathrm{MeV} /$ nucleon. Since it will be routinely achievable to accelerate protons beyond this energy at fast ignition relevant facilities, the deuterium thickness in this scheme could be tailored such that the maximum proton energy is fixed to $27 \mathrm{MeV}$ with the remaining laser energy converted into lower energy $\mathrm{MeV}$ deuterons, to provide a boost to the later time heating. As simulations show that the laser to $\mathrm{MeV}$ ion conversion efficiency is constant $( \pm 6 \%)$ as a function of the deuterium thickness up to $40 \mathrm{~nm}$, such a tuning of the spectrum should be beneficial as the laser energy is carried by ions of more favorable energy range.

Further, this acceleration scheme is compatible with existing techniques that could be utilized for global enhancement of maximum energy and conversion efficiency of both ion species [29-32], beyond those demonstrated in this proof of principle investigation.

Data associated with research published in this Letter can be accessed in Ref. [41].

The authors gratefully acknowledge the expert assistance of the CLF Vulcan operations and technical support teams.
We also gratefully acknowledge funding from EPSRC Grants No. EP/J002550/1, EP/J003832/1, No. EP/ K022415/1, No. EP/R006202/1, and NSFC Grant No. 11520101003. The EPOCH code used in this research was developed under EPSRC Grants No. EP/G054940/1, No. EP/G055165/1, and No. EP/G056803/1 and computing resources were provided by STFC Scientific Computing Department's SCARF cluster.

*graeme.scott@stfc.ac.uk david.neely@stfc.ac.uk

[1] H. Daido, M. Nishiuchi, and A. Pirozhkov, Rep. Prog. Phys. 75, 056401 (2012).

[2] A. Macchi, M. Borghesi, and M. Passoni, Rev. Mod. Phys. 85, 751 (2013).

[3] S. C. Wilks, A. B. Langdon, T. E. Cowan, M. Roth, M. Singh, S. Hatchett, M. H. Key, D. Pennington, A. MacKinnon, and R. A. Snavely, Phys. Plasmas 8, 542 (2001).

[4] P. Mora, Phys. Rev. Lett. 90, 185002 (2003).

[5] P. Mora, Phys. Rev. E 72, 056401 (2005).

[6] T. Grismayer and P. Mora, Phys. Plasmas 13, 032103 (2006).

[7] J. Schreiber et al., Phys. Rev. Lett. 97, 045005 (2006).

[8] T. Zh. Esirkepov et al., Phys. Rev. Lett. 89, 175003 (2002).

[9] V. T. Tikhonchuk, A. A Andreev, S. G Bochkarev, and V. Yu Bychenkov, Plasma Phys. Controlled Fusion 47, B869 (2005).

[10] A. P. L. Robinson, A. R. Bell, and R. J. Kingham, Phys. Rev. Lett. 96, 035005 (2006).

[11] A. P. L. Robinson, D. Neely, P. McKenna, and R. G. Evans, Plasma Phys. Controlled Fusion 49, 373 (2007).

[12] A. P. L. Robinson and P. Gibbon, Phys. Rev. E 75, 015401 (R) (2007).

[13] B. M. Hegelich, B. J. Albright, J. Cobble, K. Flippo, S. Letzring, M. Paffett, H. Ruhl, J. Schreiber, R. K. Schulze, and J. C. Fernández, Nature (London) 439, 441 (2006).

[14] H. Schwoerer, S. Pfotenhauer, O. Jäckel, K.-U. Amthor, B. Liesfeld, W. Ziegler, R. Sauerbrey, K. W. D. Ledingham, and T. Esirkepov, Nature (London) 439, 445 (2006).

[15] S. M. Pfotenhauer et al., New J. Phys. 10, 033034 (2008).

[16] S. Ter-Avetisyan, M. Schnürer, P. V. Nickles, M. Kalashnikov, E. Risse, T. Sokollik, W. Sandner, A. Andreev, and V. Tikhonchuk, Phys. Rev. Lett. 96, 145006 (2006).

[17] K. Markey et al., Phys. Rev. Lett. 105, 195008 (2010).

[18] F. Dollar et al., Phys. Rev. Lett. 107, 065003 (2011).

[19] H. Powell et al., New J. Phys. 17, 103033 (2015).

[20] N. P. Dover et al., New J. Phys. 18, 013038 (2016).

[21] H. Padda et al., Phys. Plasmas 23, 063116 (2016).

[22] S. Astbury et al., J. Phys. Conf. Ser. 713, 012006 (2016).

[23] T. D. Arber et al., Plasma Phys. Controlled Fusion 57, 113001 (2015).

[24] See Supplemental Material at http://link.aps.org/ supplemental/10.1103/PhysRevLett.120.204801 for a discussion of the role of the target composition on this acceleration mechanism.

[25] D. C. Carroll et al., Phys. Rev. E 76, 065401(R) (2007). 
[26] E. Brambrink, J. Schreiber, T. Schlegel, P. Audebert, J. Cobble, J. Fuchs, M. Hegelich, and M. Roth, Phys. Rev. Lett. 96, 154801 (2006).

[27] C. N. Danson et al., Nucl. Fusion 44, S239 (2004).

[28] A. Alejo et al., Rev. Sci. Instrum. 85, 093303 (2014).

[29] L. Robson et al., Nat. Phys. 3, 58 (2007).

[30] J. Fuchs et al., Nat. Phys. 2, 48 (2006).

[31] D. Neely, P. Foster, A. Robinson, F. Lindau, O. Lundh, A. Persson, C.-G. Wahlström, and P. McKenna, Appl. Phys. Lett. 89, 021502 (2006).

[32] C. M. Brenner et al., Appl. Phys. Lett. 104, 081123 (2014).
[33] A. G. Krygier et al., Phys. Plasmas 22, 053102 (2015).

[34] C. P. J. Barty et al., Nucl. Fusion 44, S266 (2004).

[35] Y. Kitagawa et al., Phys. Rev. Lett. 114, 195002 (2015).

[36] N. Blanchot et al., Opt. Express 25, 16957 (2017).

[37] A. Morace et al., Nucl. Fusion 57, 126018 (2017).

[38] M. Roth et al., Phys. Rev. Lett. 86, 436 (2001).

[39] M. Temporal, J. J. Honrubia, and S. Atzeni, Phys. Plasmas 9, 3098 (2002).

[40] D.-X. Liu, W. Hong, L.-Q. Shan, S.-C. Wu, and Y.-Q. Gu, Plasma Phys. Controlled Fusion 53, 035022 (2011).

[41] http://dx.doi.org/10.5286/edata/713. 